\title{
Migraine and risk of cardiovascular diseases: Danish population based matched cohort study
}

\author{
Kasper Adelborg, ${ }^{1}$ Szimonetta Komjáthiné Szépligeti, ${ }^{1}$ Louise Holland-Bill,, ${ }^{1}$ Vera Ehrenstein, ${ }^{1}$ \\ Erzsébet Horváth-Puhó, ${ }^{1}$ Victor W Henderson, ${ }^{1,2,3}$ Henrik Toft Sørensen ${ }^{1,2}$
}

${ }^{1}$ Department of Clinical

Epidemiology, Aarhus University

Hospital, Denmark

${ }^{2}$ Department of Health Research and Policy (Epidemiology),

Stanford University, Stanford,

CA, USA

${ }^{3}$ Department of Neurology and Neurological Sciences, Stanford University, Stanford, CA, USA

Correspondence to: $\mathrm{K}$ Adelborg kade@clin.au.dk

Additional material is published online only. To view please visit the journal online.

Cite this as: $B M J$ 2018;360:k96 http://dx.doi.org/10.1136/bmj.k96

Accepted: 12 December 2017

\section{ABSTRACT}

OBJECTIVE

To examine the risks of myocardial infarction, stroke (ischaemic and haemorrhagic), peripheral artery disease, venous thromboembolism, atrial fibrillation or atrial flutter, and heart failure in patients with migraine and in a general population comparison cohort.

DESIGN

Nationwide, population based cohort study.

SETTING

All Danish hospitals and hospital outpatient clinics from 1995 to 2013.

PARTICIPANTS

51032 patients with migraine and 510320 people from the general population matched on age, sex, and calendar year.

\section{MAIN OUTCOME MEASURES}

Comorbidity adjusted hazard ratios of cardiovascular outcomes based on Cox regression analysis.

RESULTS

Higher absolute risks were observed among patients with incident migraine than in the general population across most outcomes and follow-up periods. After 19 years of follow-up, the cumulative incidences per 1000 people for the migraine cohort compared with the general population were $25 v 17$ for myocardial infarction, $45 v 25$ for ischaemic stroke, $11 v 6$ for haemorrhagic stroke, $13 v 11$ for peripheral artery disease, $27 v 18$ for venous thromboembolism, 47 $v 34$ for atrial fibrillation or atrial flutter, and $19 v$ 18 for heart failure. Correspondingly, migraine was positively associated with myocardial infarction (adjusted hazard ratio 1.49, 95\% confidence interval 1.36 to 1.64$)$, ischaemic stroke $(2.26,2.11$ to 2.41$)$, and haemorrhagic stroke $(1.94,1.68$ to 2.23$)$, as well as venous thromboembolism $(1.59,1.45$ to 1.74$)$

\section{WHAT IS ALREADY KNOWN ON THIS TOPIC}

Migraine is associated with ischaemic stroke and myocardial infarction However, the associations between migraine and other cardiovascular diseases and risks in subgroups of patients are less well established

\section{WHAT THIS STUDY ADDS}

This nationwide population based cohort study showed that migraine was associated with increased risks of several cardiovascular diseases, particularly stroke, in the first year after diagnosis, which persisted in the long term

The associations were stronger in patients with aura than in those without aura and in women than in men

The absolute risks for cardiovascular outcomes, however, were low, as expected given the young age of the study population

and atrial fibrillation or atrial flutter $(1.25,1.16$ to 1.36). No meaningful association was found with peripheral artery disease (adjusted hazard ratio 1.12, 0.96 to 1.30$)$ or heart failure $(1.04,0.93$ to 1.16$)$. The associations, particularly for stroke outcomes, were stronger during the short term (0-1 years) after diagnosis than the long term (up to 19 years), in patients with aura than in those without aura, and in women than in men. In a subcohort of patients, the associations persisted after additional multivariable adjustment for body mass index and smoking.

\section{CONCLUSIONS}

Migraine was associated with increased risks of myocardial infarction, ischaemic stroke, haemorrhagic stroke, venous thromboembolism, and atrial fibrillation or atrial flutter. Migraine may be an important risk factor for most cardiovascular diseases.

\section{Introduction}

Migraine is a common primary headache disorder, characterised by recurrent episodes of neurological, gastrointestinal, and autonomic symptoms, alone or in combination. ${ }^{12}$ Migraine affects nearly $15 \%$ of the population or about one billion people worldwide. ${ }^{3}$ The lifetime prevalence of migraine is $10-20 \%$, depending on the case definition and on the age and sex distribution of the study population. ${ }^{14}$ After puberty, the incidence of migraine increases, and it does so more rapidly in women than in men. ${ }^{15}$ Approximately $90 \%$ of all patients have their first attack before the age of 50 years. ${ }^{46}$ As a leading cause of disability, ${ }^{37}$ migraine has considerable impact on quality of life and imposes a substantial burden on society.

Migraine is associated with ischaemic stroke and ischaemic heart disease, particularly among women and among migraine patients with aura. ${ }^{8} 10$ Potential underlying mechanisms include endothelial dysfunction, hypercoagulability, platelet aggregation, vasospasm, cardiovascular risk factors, paradoxical embolism, spreading depolarisation, shared genetic risk, use of non-steroidal anti-inflammatory drugs, and immobilisation. ${ }^{11-19}$ Most of these mechanisms may also contribute to the risk of other cardiovascular events; however, convincing epidemiological evidence is lacking for some of these. To improve the understanding of the cardiovascular morbidity associated with migraine, we examined the risks of myocardial infarction, ischaemic and haemorrhagic stroke, peripheral artery disease, venous thromboembolism, atrial fibrillation or atrial flutter, and heart failure in patients with a first time diagnosis of migraine and compared with the general population. We also evaluated whether the associations vary by 
migraine aura status or by sex. Such data will help to guide interventions to reduce risks and mitigate disparities.

\section{Methods}

\section{Setting and design}

We did a population based matched cohort study based on routinely and prospectively collected data from Danish administrative and medical registries (catchment dynamic population about six million inhabitants). ${ }^{20}$ A national health insurance programme ensures tax supported healthcare for the entire Danish population. The unique civil registration number, assigned to all Danish residents at birth or immigration, allows exact linkage of data from all registries, after which data are anonymised. ${ }^{20}$ The registries capture dispensings from outpatient pharmacies prescribed by general practitioners but do not capture diagnoses or other clinical data from general practice.

\section{Migraine cohort}

We included all patients with a first time primary or secondary diagnosis of migraine, as recorded in the Danish National Patient Registry (DNPR) from 1 January 1995 to 30 November 2013. The DNPR contains records on hospital admissions since 1977 and on visits to hospital outpatient clinics and emergency departments since 1995. Diagnoses are coded according to the international classification of diseases, 8th revision (ICD-8) through 1993 and the 10th revision (ICD-10) thereafter. $^{21}$ We used all recorded inpatient, outpatient, and emergency department diagnoses to identify patients with migraine. The admission date for the first contact associated with a migraine diagnosis defined the "index date." We excluded patients with a migraine diagnosis before 1995 or with previous myocardial infarction, stroke, peripheral artery disease, venous thromboembolism, atrial fibrillation or atrial flutter, or heart failure.

\section{General population comparison cohort}

From the Danish Civil Registration System, we assembled a general population comparison cohort. ${ }^{20}$ For each patient with migraine, we randomly sampled 10 people who were migraine-free and alive on the index date, matched, with replacement, on exact age in years, sex, and calendar year. The general population comparison cohort was sampled using the same exclusion criteria as the migraine cohort (that is, no previous cardiovascular events). People diagnosed as having migraine during the follow-up remained in the general population comparison cohort, to avoid informative censoring.

\section{Cardiovascular outcomes}

The incident outcomes included myocardial infarction, ischaemic and haemorrhagic stroke, peripheral artery disease, venous thromboembolism, atrial fibrillation or atrial flutter, and heart failure and were ascertained using all available inpatient and outpatient diagnoses recorded in the DNPR. To examine the underlying mechanism, we separately analysed the risk of deep vein thrombosis, pulmonary embolism, provoked venous thromboembolism (defined on the basis of a diagnosis of malignancy any time before the venous thromboembolism diagnosis, or pregnancy, trauma, or surgery 90 days before the diagnosis), and unprovoked venous thromboembolism. Registration of cardiovascular diagnoses in the DNPR are accurate, with validation studies consistently reporting positive predictive values above $80 \%$ for most conditions. ${ }^{2122}$

\section{Covariables}

Using the full hospital history (inpatient and outpatient diagnoses) recorded in the DNPR before the index date, weobtained information on the following cardiovascular risk factors: diabetes, obesity, hyperlipidaemia, hypercholesterolaemia, hypertension, valvular heart disease, chronic obstructive pulmonary disease (as an indicator of chronic smoking), renal failure, liver disease, cancer, alcoholism related disorders, and diseases of the thyroid. ${ }^{23}$

\section{Statistical analyses for main analysis}

We tabulated the distributions of the covariables for the migraine cohort overall, in migraine patients with and without aura recorded with specific ICD-10 codes, and for the general population comparison cohort. We followed both cohorts from the index date until the date of myocardial infarction, ischaemic stroke, haemorrhagic stroke, peripheral artery disease, venous thromboembolism (deep vein thrombosis and pulmonary embolisms separately), atrial fibrillation or atrial flutter, heart failure, emigration, death, or end of follow-up (30 November 2013), whichever came first. After an initial event, we continued to follow patients for subsequent other cardiovascular events to avoid informative censoring coupled with the intent to understand the full spectrum and extent of cardiovascular morbidity associated with migraine. We calculated the 0-1 year, $>1-5$ years, and >5-19 years cumulative incidence per 1000 people for each outcome, accounting for the competing risk of death. Correspondingly, we used matching factors stratified (conditional) Cox proportional hazards regression to estimate hazard ratios, adjusting for the categorical comorbidities listed above as covariables. Results were stratified by presence of aura, sex, age groups, type of diagnosis (primary/secondary; inpatient/outpatient/ emergency department), use of non-steroidal antiinflammatory drugs, use of migraine specific drugs (triptans or ergotamine), and number of cardiovascular risk factors $(0,1, \geq 2)$. Using log minus log plots, we found no deviations from the proportionality of hazard assumption in the analysed follow-up periods. We present all estimates of association with 95\% confidence intervals.

\section{Sensitivity analyses}

We did six sensitivity analyses. Firstly, because patients with migraine treated exclusively by a general 
practitioner are not captured in the DNPR, we repeated the analyses using prescription data from the National Health Service Prescription Database (data available from July 2004 onwards) to identify patients in the primary healthcare sector. ${ }^{24}$ We assembled a cohort of patients with at least two redeemed prescriptions for migraine specific treatment (triptans or ergotamine) or an ICD code for migraine. The index date was the date of the second redeemed prescription for or an ICD code for migraine, whichever came first. Secondly, for the stroke outcome, we did an analysis requiring a 14 day window between migraine diagnosis and stroke diagnosis, to reduce potential misclassification of stroke as migraine (start of follow-up on day 14). Thirdly, to improve the specificity of the stroke diagnosis, we used a stricter definition of stroke, requiring a record of a computed tomography scan or magnetic resonance imaging scan of the brain during the incident hospital contact with stroke. This analysis was based on patients diagnosed as having migraine from 1 January 2000 onwards, when data on computed tomography and magnetic resonance imaging scans became reportable to the DNPR. "Unspecified stroke"” diagnoses were classified in the main analysis as ischaemic in origin, because most of these are ischaemic strokes. ${ }^{25}$ In a fourth sensitivity analyses, we repeated the analysis with separate assessment of specified ischaemic stroke and unspecified stroke to test the robustness of this approach.

Fifthly, as several drugs may confound or mediate the association between migraine and cardiovascular events, we repeated the main analysis with additional adjustment for use of $\beta$ blockers, angiotensin converting enzyme inhibitors/angiotensin II receptor blockers, diuretics, vitamin $\mathrm{K}$ antagonists, aspirin, clopidogrel, statins, non-steroidal anti-inflammatory drugs, opioids, antipsychotics, and sex hormones (except oral contraceptives, which are not reimbursed and are therefore not captured in the Danish Health Service Prescription Database) within 90 days preceding the migraine diagnosis, using data on redeemed prescriptions. ${ }^{24}$ Sixthly, to compensate for lack of data on smoking and body mass index, we identified and characterised a subcohort of female migraine patients who were also registered in the Danish Medical Birth Registry when giving birth and for whom data on smoking and pre-pregnancy body mass index before the migraine admission date were available from 2004 onwards. ${ }^{26}$ For this subcohort, we randomly sampled up to 10 women from the Danish Medical Birth Registry matched for age and conception period. We repeated the analyses in these cohorts while adjusting for smoking (yes/no) and body mass index $(<18.5,18.5-24,25-29$, and $\geq 30)$. The ICD codes used in the study are provided in supplementary tables A and B. We used SAS version 9.4 for all analyses.

\section{Patient involvement}

No patients were involved in setting the research question or the outcome measures, nor were they involved in developing plans for design or implementation of the study. No patients were asked to advise on interpretation or writing up of results. There are no plans to disseminate the results of the research to study participants or the relevant patient community.

\section{Results}

The migraine cohort included 51032 people and the matched general population cohort 510320 . Median age at diagnosis of migraine was 35 (interquartile range $22-47$ ) years, and $71 \%$ of the study population were women. The migraine cohort had a slightly higher burden of cardiovascular risk factors and comorbidity at index date (table 1).

\section{Migraine and cardiovascular disease risk}

In the migraine cohort, 2451 patients had one cardiovascular event and 575 patients had more than one cardiovascular event. The cumulative incidences per 1000 people after 19 years of follow-up for the migraine cohort compared with the general population were 25 versus 17 for myocardial infarction, 45 versus 25 for ischaemic stroke, 11 versus six for haemorrhagic stroke, 13 versus 11 for peripheral artery disease, 27 versus 18 for venous thromboembolism, 47 versus

Table 1 | Characteristics of migraine and matched general population comparison cohorts. Values are numbers (percentages) unless stated otherwise

\begin{tabular}{|c|c|c|c|c|}
\hline Characteristics & $\begin{array}{l}\text { Migraine } \\
\text { cohort }(n=51032)\end{array}$ & 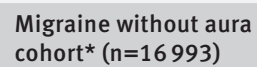 & 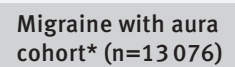 & $\begin{array}{l}\text { General population comparison } \\
\text { cohort }(n=510320)\end{array}$ \\
\hline Median (interquartile range) age, years & $35(22-47)$ & $36(23-47)$ & $34(24-46)$ & $35(22-47)$ \\
\hline Men & $14980(29.4)$ & $4515(26.6)$ & $3749(28.7)$ & $149800(29.4)$ \\
\hline Diabetes & $699(1.4)$ & $227(1.3)$ & $153(1.2)$ & $5863(1.1)$ \\
\hline Obesity & $1639(3.2)$ & $537(3.2)$ & $450(3.4)$ & $12821(2.5)$ \\
\hline Hyperlipidaemia & $93(0.2)$ & $23(0.1)$ & $24(0.2)$ & $471(0.1)$ \\
\hline Hypercholesterolaemia & $375(0.7)$ & $103(0.6)$ & $118(0.9)$ & $1928(0.4)$ \\
\hline Hypertension & $1547(3.0)$ & $498(2.9)$ & $398(3.0)$ & $9104(1.8)$ \\
\hline Valvular heart disease & $202(0.4)$ & $50(0.3)$ & $81(0.6)$ & $819(0.2)$ \\
\hline Chronic obstructive pulmonary disease & 649 (1.3) & $194(1.1)$ & $165(1.3)$ & $4168(0.8)$ \\
\hline Renal failure & $118(0.2)$ & $45(0.3)$ & $18(0.1)$ & $655(0.1)$ \\
\hline Liver disease & $239(0.5)$ & $82(0.5)$ & $48(0.4)$ & $1701(0.3)$ \\
\hline Alcoholism related disease & $643(1.3)$ & $174(1.0)$ & $154(1.2)$ & $5396(1.1)$ \\
\hline Cancer & $1237(2.4)$ & $400(2.4)$ & $309(2.4)$ & $10808(2.1)$ \\
\hline Diseases of thyroid & $1494(2.9)$ & $502(3.0)$ & $428(3.3)$ & $11235(2.2)$ \\
\hline
\end{tabular}

*Patients with available information on aura status. 

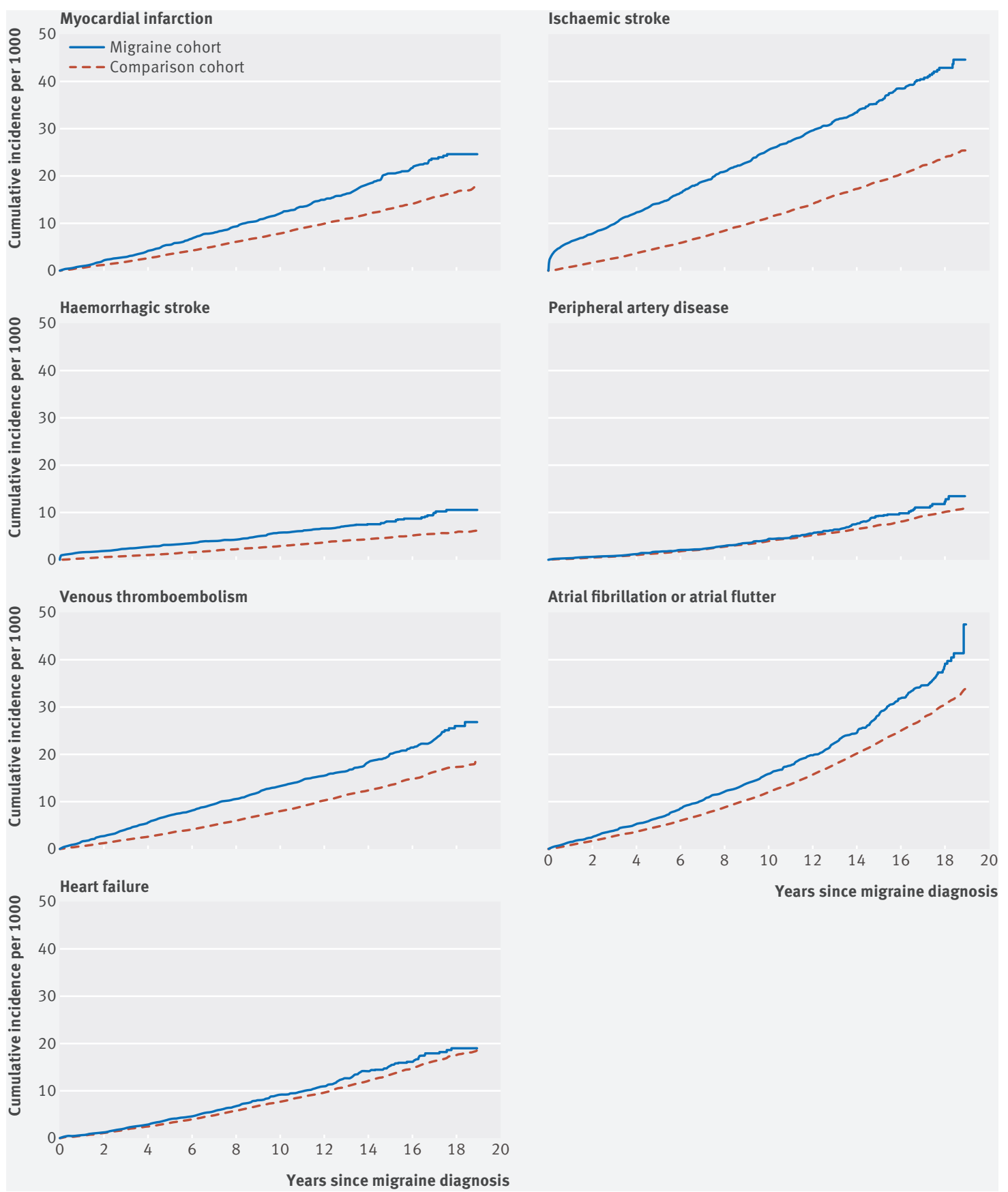

Fig 1 | Cumulative incidence of cardiovascular events in migraine and general population comparison cohorts

34 for atrial fibrillation or atrial flutter, and 19 versus 18 for heart failure (fig 1). After adjustment for the covariables, migraine was associated with myocardial infarction (adjusted hazard ratio 1.49, 95\% confidence interval 1.36 to 1.64$)$, ischaemic stroke $(2.26,2.11$ to 2.41), and haemorrhagic stroke (1.94, 1.68 to 2.23$)$, as well as venous thromboembolism $(1.59,1.45$ to 1.74$)$ and atrial fibrillation or atrial flutter $(1.25,1.16$ to 1.36) (fig 2). We found no association with peripheral artery disease (adjusted hazard ratio $1.12,0.96$ to 1.30) or heart failure (1.04, 0.93 to 1.16$)$. The risk of cardiovascular disease was highest during the first year, with an eightfold increased risk of ischaemic and haemorrhagic stroke and an approximately twofold increased risk of myocardial infarction, venous thromboembolism, and atrial fibrillation or atrial flutter (fig 2). During $>1-5$ years and $>5-19$ years, migraine remained associated with myocardial infarction (1.5 fold), ischaemic stroke (1.6-2.1 fold), haemorrhagic stroke (1.4 fold), venous thromboembolism (1.3-2.0 fold), and atrial fibrillation or atrial flutter (1.2 fold) (fig 2). The risks were similar for deep vein thrombosis and pulmonary embolism and were slightly higher for unprovoked venous thromboembolism than for provoked venous thromboembolism (supplementary table C). 


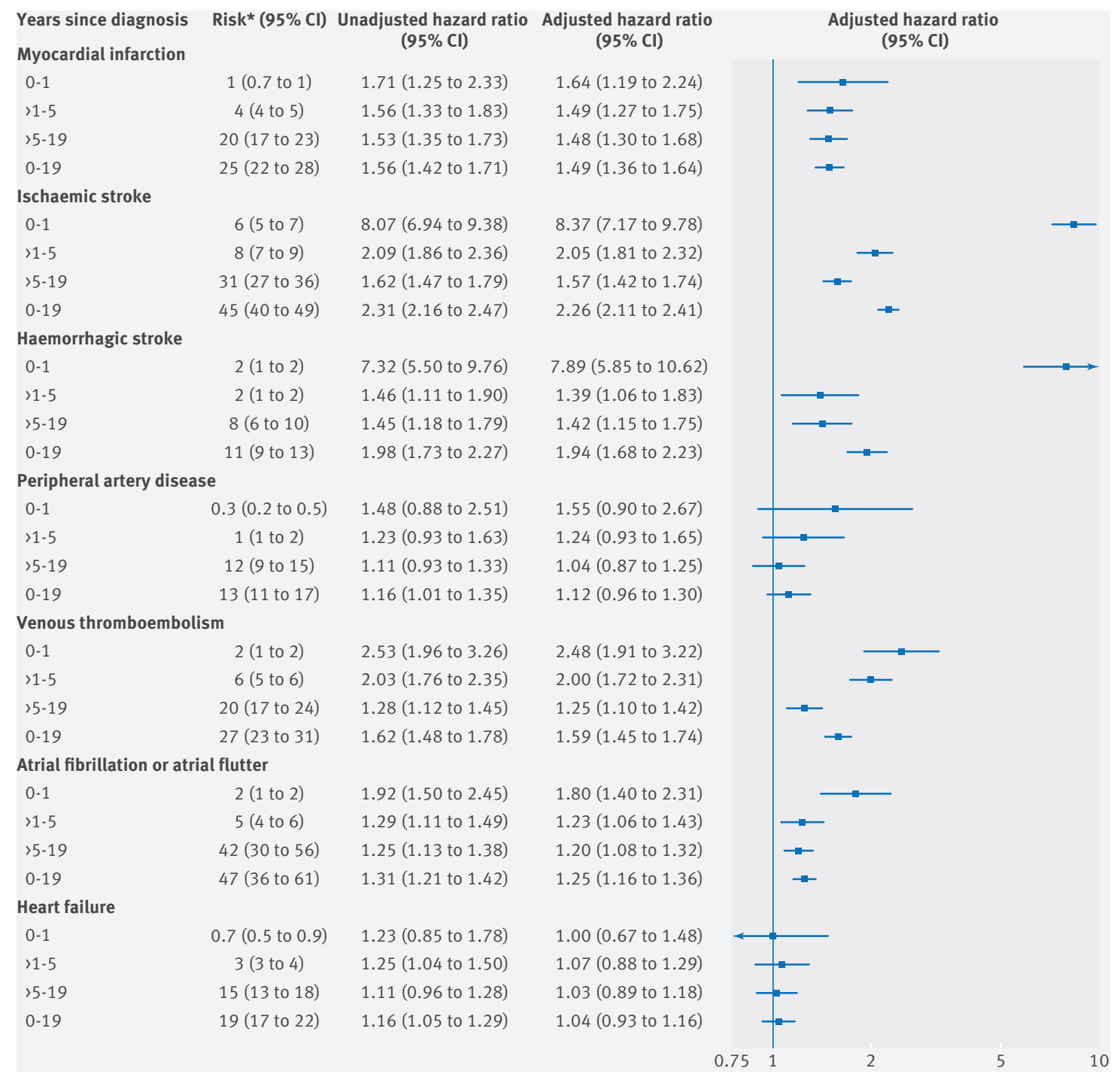

Fig 2 | Risk of cardiovascular events among patients with migraine relative to general population comparison cohort, by follow-up interval since migraine diagnosis/index date. *Cumulative incidence per 1000 migraine patients. Unadjusted hazard ratios: controlled for age, sex, and calendar year. Adjusted hazard ratios: controlled for age, sex, and calendar year and adjusted for covariables in table 1

\section{Subgroup analyses}

Information on aura status was available for 30069 migraine patients $(59 \%$ of the migraine cohort). In general, the adjusted hazard ratios were higher for migraine with aura than for migraine without aura for all outcomes except for venous thromboembolism and heart failure (table 2). Migraine with aura was associated with long term risks of myocardial infarction, ischaemic stroke, haemorrhagic stroke, and venous thromboembolism.

The associations were somewhat stronger among women than among men but persisted for both sexes (table 3) and generally attenuated with increasing age (supplementary tables D-F). The associations were in most cases stronger in patients with inpatient or emergency department diagnoses than in outpatients (supplementary table G), but they were similar for patients with primary and secondary diagnoses of migraine (supplementary table $\mathrm{H}$ ). Most associations persisted in non-users of non-steroidal anti-inflammatory drugs and non-users of migraine specific drugs (supplementary tables I and J). The adjusted hazard ratios for ischaemic stroke were lower in users compared with non-users of non-steroidal anti-inflammatory drugs and migraine specific drugs. Although the confidence intervals were relatively wide in analyses stratified by cardiovascular risk factors, the associations were amplified in patients without cardiovascular risk factors (supplementary table K).

\section{Sensitivity analyses}

When we included prescription data in the definition of the migraine cohort ( $81 \%$ of the patients were identified through prescription data in this analysis), the associations tapered off and disappeared for $>1-5$ year myocardial infarction and 0-1 year atrial fibrillation or atrial flutter (supplementary table L). The risk of ischaemic and haemorrhagic stroke remained elevated 


\begin{tabular}{|c|c|c|c|c|}
\hline \multirow[b]{2}{*}{ Years since diagnosis } & \multicolumn{2}{|c|}{ Cumulative incidence per 1000 in migraine cohort, \% $(95 \% \mathrm{Cl})$} & \multicolumn{2}{|c|}{ Adjusted hazard ratio $(95 \% \mathrm{Cl})^{*}$} \\
\hline & Without aura & With aura & Without aura & With aura \\
\hline \multicolumn{5}{|l|}{ Myocardial infarction } \\
\hline $0-1$ & $0.55(0.28$ to 1.02$)$ & $1.04(0.59$ to 1.76$)$ & $1.07(0.52$ to 2.20$)$ & $1.95(1.05$ to 3.64$)$ \\
\hline$>1-5$ & $3.11(2.27$ to 4.20$)$ & $4.31(3.14$ to 5.82$)$ & $1.21(0.87$ to 1.68$)$ & $1.47(1.05$ to 2.05$)$ \\
\hline$>5-19$ & $16.26(12.22$ to 21.24$)$ & $22.63(16.53$ to 30.25$)$ & $1.28(0.99$ to 1.63$)$ & $1.92(1.49$ to 2.48$)$ \\
\hline $0-19$ & 19.65 (15.50 to 24.59$)$ & $27.58(21.32$ to 35.07$)$ & $1.22(1.01$ to 1.48$)$ & 1.74 (1.44 to 2.11$)$ \\
\hline \multicolumn{5}{|l|}{ Ischaemic stroke } \\
\hline $0-1$ & 2.77 (2.06 to 3.68$)$ & $7.54(6.15$ to 9.16$)$ & $4.54(3.16$ to 6.51$)$ & $10.25(7.65$ to 13.72$)$ \\
\hline$>1-5$ & $7.01(5.70$ to 8.55$)$ & $7.25(5.69$ to 9.14$)$ & $1.90(1.52$ to 2.38$)$ & $2.03(1.55$ to 2.65$)$ \\
\hline$>5-19$ & $31.77(23.20$ to 42.38$)$ & $26.82(20.00$ to 35.18$)$ & $1.45(1.20$ to 1.76$)$ & $1.52(1.21$ to 1.91$)$ \\
\hline $0-19$ & 40.85 (32.10 to 51.11) & 40.83 (33.63 to 49.02$)$ & 1.81 (1.58 to 2.06$)$ & 2.49 (2.16 to 2.86$)$ \\
\hline \multicolumn{5}{|l|}{ Haemorrhagic stroke } \\
\hline $0-1$ & $1.38(0.91$ to 2.05$)$ & 1.26 (0.76 to 2.02$)$ & 8.03 (4.57 to 14.13$)$ & 7.04 (3.58 to 13.84$)$ \\
\hline$>1-5$ & 1.36 (0.84 to 2.14$)$ & $1.30(0.72$ to 2.24$)$ & 1.30 (0.79 to 2.15$)$ & 1.13 (0.61 to 2.08 ) \\
\hline$>5-19$ & 6.25 (3.71 to 10.04$)$ & 6.54 (3.65 to 11.02$)$ & 1.15 (0.76 to 1.74$)$ & 1.52 (0.95 to 2.44$)$ \\
\hline $0-19$ & $8.90(6.14$ to 12.56$)$ & $8.99(5.87$ to 13.30$)$ & $1.70(1.31$ to 2.21$)$ & 1.82 (1.34 to 2.48$)$ \\
\hline \multicolumn{5}{|c|}{ Peripheral artery disease } \\
\hline $0-1$ & 0.24 (0.09 to 0.62$)$ & $0.40(0.16$ to 0.93$)$ & $1.10(0.37$ to 3.26$)$ & $1.62(0.62$ to 4.24$)$ \\
\hline$>1-5$ & $1.02(0.58$ to 1.72$)$ & $1.66(0.99$ to 2.66$)$ & $0.82(0.45$ to 1.47$)$ & 1.77 (1.02 to 3.07) \\
\hline$>5-19$ & $9.55(5.60$ to 15.41$)$ & $15.76(8.27$ to 27.54$)$ & $0.85(0.58$ to 1.24$)$ & $1.24(0.85$ to 1.80$)$ \\
\hline $0-19$ & 10.67 (6.66 to 16.37$)$ & 17.58 (9.96 to 28.94$)$ & $0.86(0.63$ to 1.16$)$ & $1.38(1.03$ to 1.85$)$ \\
\hline \multicolumn{5}{|c|}{ Venous thromboembolism } \\
\hline $0-1$ & 1.71 (1.17 to 2.44$)$ & 1.13 (0.66 to 1.88$)$ & $2.80(1.83$ to 4.30$)$ & 2.18 (1.19 to 3.98$)$ \\
\hline$>1-5$ & 4.66 (3.61 to 5.95$)$ & $6.22(4.77$ to 8.00$)$ & $1.60(1.22$ to 2.10$)$ & 2.25 (1.68 to 3.01$)$ \\
\hline$>5-19$ & 22.25 (15.54 to 30.88) & 18.00 (11.56 to 26.82) & 1.35 (1.08 to 1.70$)$ & $0.97(0.71$ to 1.33$)$ \\
\hline $0-19$ & $28.19(21.31$ to 36.55$)$ & $24.98(18.19$ to 33.45$)$ & 1.57 (1.34 to 1.84$)$ & $1.49(1.22$ to 1.81$)$ \\
\hline \multicolumn{5}{|c|}{ Atrial fibrillation or atrial flutter } \\
\hline $0-1$ & 0.97 (0.59 to 1.56$)$ & $2.03(1.35$ to 2.96$)$ & $1.36(0.79$ to 2.34$)$ & 2.73 (1.73 to 4.32$)$ \\
\hline$>1-5$ & 4.09 (3.12 to 5.30$)$ & $5.94(4.52$ to 7.69$)$ & 1.19 (0.89 to 1.58$)$ & $1.22(0.91$ to 1.63$)$ \\
\hline$>5-19$ & $45.65(19.26$ to 89.60$)$ & 33.04 (24.14 to 44.05$)$ & $1.13(0.92$ to 1.37$)$ & $1.21(0.97$ to 1.51$)$ \\
\hline $0-19$ & $49.92(23.14$ to 92.05$)$ & $40.33(31.26$ to 51.05$)$ & $1.16(0.99$ to 1.35$)$ & 1.31 (1.11 to 1.55$)$ \\
\hline \multicolumn{5}{|l|}{ Heart failure } \\
\hline $0-1$ & $0.67(0.36$ to 1.18$)$ & $0.73(0.37$ to 1.36$)$ & 1.06 (0.49 to 2.27$)$ & $1.28(0.60$ to 2.73$)$ \\
\hline$>1-5$ & $3.48(2.57$ to 4.65$)$ & $2.98(2.03$ to 4.28$)$ & $1.28(0.91$ to 1.80$)$ & $1.09(0.73$ to 1.64$)$ \\
\hline$>5-19$ & $15.60(11.35$ to 20.97$)$ & 11.74 (8.19 to 16.40$)$ & 1.24 (0.96 to 1.62$)$ & $0.96(0.69$ to 1.33$)$ \\
\hline $0-19$ & 19.49 (15.09 to 24.78$)$ & $15.26(11.50$ to 19.90$)$ & $1.24(1.02$ to 1.51$)$ & $1.02(0.80$ to 1.30$)$ \\
\hline
\end{tabular}

but became attenuated in response to requirement for a minimum of 14 days between diagnoses of migraine and stroke. Requirement for a computed tomography or magnetic resonance imaging scan for stroke definition and separate assessment of unspecified stroke and specified ischaemic stroke yielded results similar to the main analyses (supplementary table M). Additional adjustment for drugs did not materially affect the associations (supplementary table $\mathrm{N}$ ). In the subset of women with $(n=4772)$ and without $(n=47715)$ migraine from the Danish Medical Birth Registry, the overall associations persisted after adjustment for smoking and body mass index. The 0-11 year adjusted hazard ratios were 3.10 (0.92 to 10.43) for myocardial infarction, 9.87 (5.62 to 17.34 ) for ischaemic stroke, 1.81 (0.52 to 6.30) for haemorrhagic stroke, 1.78 (0.85 to 3.73 ) for venous thromboembolism, and 4.13 (1.53 to 11.15) for atrial fibrillation or atrial flutter. Owing to very few events in this population, the adjusted hazard ratios for peripheral artery disease and heart failure could not be estimated. The baseline characteristics of the migraine patients from the Danish Medical Birth Registry were comparable to those of the overall migraine cohort (supplementary table $\mathrm{O}$ ).

\section{Discussion}

In this study, migraine was associated with increased risks of several cardiovascular diseases in the short term, which persisted in the long term. The associations were stronger in patients with aura than in those without aura and in women than in men. The absolute risks for all cardiovascular outcomes, however, were low, which is expected given the young age of the study population.

\section{Strengths and limitations of study}

Strengths of our study are the population based design and long term complete follow-up, both of which reduce the risk of selection bias arising from residence, insurance, income, or age dependent inclusion or informative loss to follow-up. The large sample size allowed us to study specific cardiovascular diseases. The positive predictive value in the DNPR is high for diagnoses of myocardial infarction (97\%), ischaemic stroke (97\%), peripheral artery disease (91\%), venous thromboembolism $(88 \%)$, and atrial fibrillation or atrial flutter (95\%), but it is somewhat lower for haemorrhagic stroke $(60-70 \%)$ and heart failure $(80 \%) .^{21}{ }^{22}$ The validity of the migraine diagnosis in 


\begin{tabular}{|c|c|c|c|c|}
\hline \multirow[b]{2}{*}{ Years since diagnosis } & \multicolumn{2}{|c|}{ Cumulative incidence per 1000 in migraine cohort, $\%(95 \% \mathrm{Cl})$} & \multicolumn{2}{|c|}{ Adjusted hazard ratio $(95 \% \mathrm{Cl})^{*}$} \\
\hline & Women & Men & Women & Men \\
\hline \multicolumn{5}{|l|}{ Myocardial infarction } \\
\hline $0-1$ & $0.74(0.50$ to 1.08$)$ & $1.46(0.94$ to 2.20$)$ & $1.62(1.05$ to 2.49$)$ & $1.74(1.08$ to 2.80$)$ \\
\hline$>1-5$ & $3.76(3.09$ to 4.55$)$ & $6.17(4.88$ to 7.72$)$ & 1.60 (1.29 to 1.98$)$ & $1.37(1.07$ to 1.76$)$ \\
\hline$>5-19$ & 16.08 (13.22 to 19.38$)$ & 27.72 (22.34 to 33.98) & 1.51 (1.27 to 1.78$)$ & $1.45(1.20$ to 1.76$)$ \\
\hline $0-19$ & $20.27(17.33$ to 23.56$)$ & $34.63(29.11$ to 40.85$)$ & $1.54(1.36$ to 1.75$)$ & $1.44(1.24$ to 1.66$)$ \\
\hline \multicolumn{5}{|l|}{ Ischaemic stroke } \\
\hline $0-1$ & $5.61(4.87$ to 6.43$)$ & $7.27(5.99$ to 8.75$)$ & $7.40(6.13$ to 8.93$)$ & $11.97(8.95$ to 16.02$)$ \\
\hline$>1-5$ & $8.28(7.27$ to 9.41$)$ & 8.09 (6.58 to 9.87$)$ & 2.19 (1.89 to 2.53$)$ & $1.81(1.45$ to 2.27$)$ \\
\hline$>5-19$ & $31.26(26.61$ to 36.45$)$ & $31.18(23.69$ to 40.22$)$ & $1.63(1.44$ to 1.84$)$ & $1.44(1.19$ to 1.73$)$ \\
\hline $0-19$ & $44.22(39.46$ to 49.36$)$ & $45.45(37.71$ to 54.21$)$ & $2.29(2.11$ to 2.49$)$ & 2.17 (1.92 to 2.45$)$ \\
\hline \multicolumn{5}{|l|}{ Haemorrhagic stroke } \\
\hline $0-1$ & $1.75(1.36$ to 2.24$)$ & $1.28(0.80$ to 1.98$)$ & $8.13(5.76$ to 11.48$)$ & $7.73(4.15$ to 14.38$)$ \\
\hline$>1-5$ & 1.55 (1.13 to 2.08$)$ & 1.66 (1.05 to 2.53$)$ & $1.29(0.92$ to 1.80$)$ & 1.64 (1.01 to 2.65$)$ \\
\hline$>5-19$ & $7.22(5.28$ to 9.70$)$ & $8.15(5.13$ to 12.44$)$ & $1.32(1.02$ to 1.70$)$ & 1.71 (1.17 to 2.50$)$ \\
\hline $0-19$ & 10.39 (8.33 to 12.83$)$ & $10.92(7.71$ to 15.07$)$ & 1.88 (1.59 to 2.22$)$ & $2.13(1.64$ to 2.76$)$ \\
\hline \multicolumn{5}{|c|}{ Peripheral artery disease } \\
\hline $0-1$ & $0.29(0.15$ to 0.52$)$ & $0.42(0.18$ to 0.89$)$ & 1.35 (0.68 to 2.68$)$ & $1.78(0.70$ to 4.52$)$ \\
\hline$>1-5$ & $1.43(1.04$ to 1.95$)$ & 1.38 (0.83 to 2.22$)$ & $1.23(0.87$ to 1.73$)$ & 1.28 (0.74 to 2.19$)$ \\
\hline$>5-19$ & $11.30(8.25$ to 15.17$)$ & 13.26 (8.74 to 19.39$)$ & $1.00(0.80$ to 1.26$)$ & $1.14(0.84$ to 1.56$)$ \\
\hline $0-19$ & 12.83 (9.75 to 16.61$)$ & $14.79(10.23$ to 20.76$)$ & $1.08(0.90$ to 1.29$)$ & $1.21(0.94$ to 1.57$)$ \\
\hline \multicolumn{5}{|c|}{ Venous thromboembolism } \\
\hline $0-1$ & 1.62 (1.24 to 2.09$)$ & 1.24 (0.77 to 1.93$)$ & 2.41 (1.78 to 3.25$)$ & 2.72 (1.59 to 4.66$)$ \\
\hline$>1-5$ & $6.49(5.60$ to 7.49$)$ & 3.65 (2.67 to 4.90$)$ & 2.10 (1.78 to 2.47$)$ & 1.66 (1.19 to 2.33$)$ \\
\hline$>5-19$ & 20.58 (16.54 to 25.30$)$ & 19.11 (13.67 to 26.03$)$ & 1.25 (1.07 to 1.45$)$ & 1.24 (0.96 to 1.61$)$ \\
\hline $0-19$ & $28.20(24.05$ to 32.84$)$ & 23.55 (17.98 to 30.29$)$ & $1.63(1.47$ to 1.80$)$ & $1.48(1.23$ to 1.79$)$ \\
\hline \multicolumn{5}{|c|}{ Atrial fibrillation or atrial flutter } \\
\hline $0-1$ & $1.24(0.91$ to 1.66$)$ & $2.28(1.61$ to 3.18$)$ & $1.73(1.24$ to 2.42$)$ & 1.89 (1.28 to 2.79$)$ \\
\hline$>1-5$ & $4.46(3.73$ to 5.30$)$ & $6.63(5.28$ to 8.25$)$ & $1.26(1.04$ to 1.53$)$ & $1.18(0.93$ to 1.51$)$ \\
\hline$>5-19$ & $36.20(30.30$ to 42.87$)$ & $51.53(24.34$ to 93.76$)$ & $1.33(1.18$ to 1.50$)$ & $0.97(0.80$ to 1.16$)$ \\
\hline $0-19$ & 41.16 (35.27 to 47.70$)$ & 59.06 (31.45 to 98.87$)$ & 1.34 (1.22 to 1.48$)$ & 1.11 (0.97 to 1.27$)$ \\
\hline \multicolumn{5}{|l|}{ Heart failure } \\
\hline $0-1$ & 0.75 (0.50 to 1.09$)$ & $0.41(0.18$ to 0.88$)$ & $1.28(0.82$ to 2.01$)$ & $0.53(0.22$ to 1.28$)$ \\
\hline$>1-5$ & $3.38(2.75$ to 4.14$)$ & $3.27(2.37$ to 4.44$)$ & $1.19(0.94$ to 1.51$)$ & $0.86(0.61$ to 1.21$)$ \\
\hline$>5-19$ & $13.60(10.97$ to 16.70$)$ & 19.01 (14.40 to 24.64$)$ & $1.01(0.84$ to 1.22$)$ & $1.04(0.82$ to 1.32$)$ \\
\hline $0-19$ & 17.48 (14.75 to 20.57$)$ & 22.28 (17.59 to 27.83$)$ & 1.09 (0.95 to 1.25$)$ & 0.95 (0.79 to 1.15$)$ \\
\hline
\end{tabular}

*Controlled for matching factors (age, sex, calendar year) by study design and adjusted for diabetes, obesity, hyperlipidaemia, hypercholesterolaemia, hypertension, valvular heart disease, chronic obstructive pulmonary disease, renal failure, liver disease, cancer, alcoholism related diseases, and diseases of thyroid.

the DNPR is unknown. Misclassification of non-stroke outcomes is likely to be independent of presence or absence of migraine. We cannot rule out that some stroke outcomes represented misdiagnosed migraine. In addition, surveillance bias cannot be excluded entirely; however, as we observed no association between migraine and peripheral artery disease or heart failure, surveillance bias is unlikely to explain its association with other cardiovascular diseases. Although we and adjusted the analyses for a wide range of potential confounders identified a priori on the basis of excisting literature, we cannot exclude influence of unknown or residual confounding, for example, by physical activity. Data on use of oral contraceptives were lacking; however, most associations were present in men as well as women.

\section{Comparison with other studies}

Many studies have assessed the risk of myocardial infarction in patients with migraine, drawing different conclusions. ${ }^{9}$ A 2015 meta-analysis of 15 studies reported a pooled relative risk of 1.33 (95\% confidence interval 1.08 to 1.64 ) among patients with migraine relative to those without migraine. ${ }^{9}$ Relative risks of myocardial infarction were comparable for men $(1.48,0.89$ to 2.45$)$ and women $(1.67,1.36$ to 2.06). In line with these findings, the Nurses' Health Study II of 115541 women aged 25-42 years, showed that migraine was associated with myocardial infarction (hazard ratio $1.39,1.18$ to 1.64 ), angina/ coronary revascularisation $(1.73,1.29$ to 2.32$)$, and cardiovascular mortality $(1.37,1.29$ to 2.32$){ }^{8}$

The relation between migraine and ischaemic stroke is well established. Our findings agree in part with a 2009 meta-analysis of nine studies, which reported increased risk of ischaemic stroke in patients with migraine (pooled relative risk $1.73,1.31$ to 2.29$).{ }^{10}$ In contrast with our findings, this meta-analysis suggested that an increased risk of ischaemic stroke was present only in patients with migraine with aura (relative risk $2.16,1.53$ to 3.03) but not in those without aura (1.23, 0.90 to 1.69). The relative risk was elevated to a greater extent among women $(2.08,1.13$ to 3.84$)$ than among men $(1.37,0.89$ to 2.11$)$. In a recent US study, which examined a cohort of patients who had surgery under general anaesthesia, patients with migraine had higher 30 day risk of ischaemic stroke than those without migraine (adjusted odds ratio 1.75, 1.39 to 2.21), 
suggesting that migraine should be included in the risk assessment for perioperative ischaemic stroke. ${ }^{27}$ Confirming previous findings, the associations between migraine and cardiovascular events were strongest in patients without cardiovascular risk factors. ${ }^{28}$

In general, relative risk estimates in our study were higher than those in previous studies. One explanation could be our inclusion of migraine patients diagnosed in an inpatient, emergency department, or outpatient hospital setting, indicating that we may have included more patients with severe migraine than previous studies-that is, migraine necessitating treatment in a hospital setting because of higher frequency, greater pain, or longer duration. In support of this inference, we observed attenuated risks when we redefined the migraine cohort to include patients receiving prescriptions for migraine treatments by community based general practitioners.

In accordance with our findings, another metaanalysis also pointed towards an increased risk of haemorrhagic stroke associated with migraine (pooled relative risk $1.48,1.16$ to 1.88$).{ }^{29}$ Mechanisms explaining the association may include alterations in the vessel wall in migraineurs and associated cardiovascular risk factors. Furthermore, arteriovenous malformations causing haemorrhagic stroke may mimic migraine. ${ }^{30}$

Few studies have looked at the association between migraine and venous thromboembolism, and results are conflicting. ${ }^{31} 32$ In a large Taiwanese cohort study of patients with migraine and a sex and propensity score matched comparison cohort, ${ }^{33}$ venous thromboembolism was not increased in migraine patients overall but only in those with migraine with aura. In two other studies of highly selected patients (pregnant women and adults aged 55 years or over), migraine was associated with venous thromboembolism. ${ }^{32} 34$ Our study extends the findings of previous studies by including data on provoked and unprovoked venous thromboembolism. In addition, our finding of an association between migraine and atrial fibrillation or atrial flutter and our finding of no association between migraine and heart failure and peripheral artery disease are novel.

\section{Potential biological mechanisms}

The mechanisms by which migraine might increase cardiovascular disease risk are probably multifactorial. The risk of stroke was markedly higher in the first year after the migraine diagnosis than in subsequent years; however, for most other outcomes, the hazard ratios remained relatively constant during follow-up. This may indicate that different mechanisms are involved in the link between migraine and specific cardiovascular outcomes. The prevalence of cardiovascular risk factors is higher in migraineurs than in people without migraine, ${ }^{15}$ a finding also apparent in our analysis. The prevalence of patent foramen ovale is also higher for migraine with than without aura $(47 \% \mathrm{v} 16 \%),{ }^{16}$ which may cause paradoxical embolism, leading in turn to cerebral or coronary ischaemic events. Patients with migraine often use non-steroidal anti-inflammatory drugs, which are associated with increased risks of myocardial infarction, venous thromboembolism, and atrial fibrillation or atrial flutter in patients without cardiovascular disease. ${ }^{193536}$ Although the association between migraine and ischaemic stroke was attenuated in users of migraine specific drugs, other data on the relation between migraine specific drugs and cardiovascular events are conflicting. ${ }^{37}$ Shared genetic mutations and inflammatory, vascular, endothelial, electrical/depolarising, and coagulable factors have been suggested as putative mechanisms for both migraine and stroke, ${ }^{1838}$ which may also apply to other cardiovascular outcomes. Finally, immobilisation related to migraine attacks may increase the risk of venous thromboembolism.

\section{Implications}

Although themagnitude of theincreased cardiovascular risk associated with migraine was fairly small at the individual level, it translates into a substantial increase in risk at the population level, because migraine is a common disease. The QRISK3 algorithm was recently developed and validated to predict 10 year risk of cardiovascular disease in men and women aged 25-84 years. ${ }^{39}$ For the first time, migraine was included in a cardiovascular risk stratification tool, underscoring the growing recognition of migraine as an important risk factor for arterial cardiovascular diseases to be considered in clinical practice. Our analysis extend this association to other common cardiovascular diseases.

Current migraine guidelines do not recommend use of aspirin and clopidogrel in the prophylaxis of migraine, ${ }^{4041}$ but clinicians should consider whether patients at particularly high risk of cardiovascular diseases would benefit from anticoagulant treatment. Ultimately, it will be important to determine whether prevention strategies in patients with migraine can reduce the burden of cardiovascular disease in patients with this common disorder.

\section{Conclusion}

In this nationwide cohort study, migraine was associated with an increased risk of cardiovascular disease. This suggests that migraine should be considered a potent and persistent risk factor for most cardiovascular diseases in both men and women.

Contributors: KA, SKS, LHB, VE, and HTS designed the study. EHP and HTS collected the data. KA reviewed the literature. KA, LHB, VWH, VE, EHP, and HTS directed the analyses, which were carried out by SKS. All authors participated in the discussion and interpretation of the results. KA organised the writing and wrote the initial draft. All authors critically revised the manuscript for intellectual content and approved the final version. HTS is the guarantor.

Funding: Aarhus University and the Program for Clinical Research Infrastructure (PROCRIN), established by the Lundbeck Foundation and the Novo Nordisk Foundation, funded the study. The sponsors had no role in study design, data collection, analysis or interpretation of the data, or writing of the manuscript or in the decision to submit the paper for publication. All authors had full access to the study data and had final responsibility for the decision to submit for publication. Competing interests: All authors have completed the ICMJE uniform disclosure form at http://www.icmje.org/coi_disclosure.pdf (available on request from the corresponding author) and declare: no support 
from any organisation for the submitted work other than that detailed above; no financial relationships in the previous three years with any organisations that might have an interest in the submitted work; and no other relationships or activities that could appear to have influenced the submitted work.

Ethics approval: The study was approved by the Danish Data Protection Agency (record number 2006-53-1396). According to Danish legislation, informed consent or approval from an ethical committee is not required for registry based studies.

Data sharing: No additional data available.

Transparency: The senior author, HTS, affirms that the manuscript is an honest, accurate, and transparent account of the study being reported; that no important aspects of the study have been omitted; and that any discrepancies from the study as planned (and, if relevant, registered) have been explained.

This is an Open Access article distributed in accordance with the Creative Commons Attribution Non Commercial (CC BY-NC 4.0) license, which permits others to distribute, remix, adapt, build upon this work non-commercially, and license their derivative works on different terms, provided the original work is properly cited and the use is non-commercial. See: http://creativecommons.org/licenses/ by-nc/4.0/.

1 Bigal ME, Lipton RB, Stewart WF. The epidemiology and impact of migraine. Curr Neurol Neurosci Rep 2004;4:98-104. doi:10.1007/ s11910-004-0022-8

2 Headache Classification Committee of the International Headache Society (IHS). The International Classification of Headache Disorders, 3rd edition (beta version). Cephalalgia 2013:33:629-808. doi:10.1177/0333102413485658

3 Vos T, Flaxman AD, Naghavi M, et al. Years lived with disability (YLDs) for 1160 sequelae of 289 diseases and injuries 1990-2010: a systematic analysis for the Global Burden of Disease Study 2010. Lancet 2012;380:2163-96. doi:10.1016/S0140 6736(12)61729-2

4 Lipton RB, Bigal ME. The epidemiology of migraine. Am J Med 2005;118(Suppl 1):3S-10S

5 Burch RC, Loder S, Loder E, Smitherman TA. The prevalence and burden of migraine and severe headache in the United States: updated statistics from government health surveillance studies. Headache 2015;55:21-34. doi:10.1111/head.12482

6 Rasmussen BK, Jensen R, Schroll M, Olesen J. Epidemiology of headache in a general population--a prevalence study. J Clin Epidemiol 1991;44:1147-57. doi:10.1016/0895 4356(91)90147-2

7 Leonardi M, Steiner TJ, Scher AT, Lipton RB. The global burden of migraine: measuring disability in headache disorders with WHO's Classification of Functioning, Disability and Health (ICF). J Headache Pain 2005:6:429-40. doi:10.1007/s10194-005-0252-4

8 Kurth T, Winter AC, Eliassen AH, et al. Migraine and risk of cardiovascular disease in women: prospective cohort study. BM/ 2016:353:i2610. doi:10.1136/bmj.i2610

9 Sacco S, Ornello R, Ripa P, et al. Migraine and risk of ischaemic heart disease: a systematic review and meta-analysis of observational studies. Eur / Neurol 2015;22:1001-11. doi:10.1111/ene.12701

10 Schürks M, Rist PM, Bigal ME, Buring JE, Lipton RB, Kurth T. Migraine and cardiovascular disease: systematic review and meta-analysis. BMJ 2009;339:b3914. doi:10.1136/bmj.b3914

11 Tietjen GE. Migraine as a systemic vasculopathy. Cephalalgia 2009;29:987-96. doi:10.1111/j.1468-2982.2009.01937.x

12 Lee ST, Chu K, Jung KH, et al. Decreased number and function of endothelial progenitor cells in patients with migraine. Neurology 2008;70:1510-7. doi:10.1212/01.wnl.0000294329.93565.94

13 Jesurum JT, Fuller C), Murinova N, Truva CM, Lucas SM. Aspirin's effect on platelet inhibition in migraineurs. Headache 2012;52:1207-18. doi:10.1111/j.1526-4610.2012.02143.x

14 Vanmolkot FH, Van Bortel LM, de Hoon JN. Altered arterial function in migraine of recent onset. Neurology 2007;68:1563-70. doi:10.1212/01.wnl.0000260964.28393.ed

15 Bigal ME, Kurth T, Hu H, Santanello N, Lipton RB. Migraine and cardiovascular disease: possible mechanisms of interaction. Neurology 2009;72:1864-71. doi:10.1212/ WNL.0b013e3181a71220

16 Schwerzmann M, Nedeltchev K, Lagger F, et al. Prevalence and size of directly detected patent foramen ovale in migraine with aura. Neurology 2005;65:1415-8. doi:10.1212/01. wnl.0000179800.73706.20

17 Dreier JP, Reiffurth C. The stroke-migraine depolarization continuum Neuron 2015:86:902-22. doi:10.1016/j.neuron.2015.04.004

18 Eikermann-Haerter K, Lee JH, Yuzawa I, et al. Migraine mutations increase stroke vulnerability by facilitating ischemic depolarizations. Circulation 2012;125:335-45. doi:10.1161/ CIRCULATIONAHA.111.045096
19 Trelle S, Reichenbach S, Wandel S, et al. Cardiovascular safety of non-steroidal anti-inflammatory drugs: network meta-analysis. BM/ 2011;342:c7086. doi:10.1136/bmi.c7086

20 Schmidt M, Pedersen L, Sørensen HT. The Danish Civil Registration System as a tool in epidemiology. Eur J Epidemiol 2014;29:541-9. doi:10.1007/s10654-014-9930-3

21 Schmidt M, Schmidt SA, Sandegaard JL, Ehrenstein V, Pedersen L, Sørensen HT. The Danish National Patient Registry: a review of content, data quality, and research potential. Clin Epidemiol 2015;7:449-90. doi:10.2147/CLEP.S91125

22 Sundbøll J, Adelborg K, Munch T, et al. Positive predictive value of cardiovascular diagnoses in the Danish National Patient Registry: a validation study. BMJ Open 2016;6:e012832. doi:10.1136/ bmjopen-2016-012832

23 Ochs N, Auer R, Bauer DC, et al. Meta-analysis: subclinical thyroid dysfunction and the risk for coronary heart disease and mortality. Ann Intern Med 2008;148:832-45. doi:10.7326/0003-4819-148-11 200806030-00225

24 Johannesdottir SA, Horváth-Puhó E, Ehrenstein V Schmidt M, Pedersen L, Sørensen HT. Existing data sources for clinical epidemiology: The Danish National Database of Reimbursed Prescriptions. Clin Epidemiol 2012;4:303-13. doi:10.2147/CLEP. S37587

25 Krarup LH, Boysen G, Janjua H, Prescott E, Truelsen T. Validity of stroke diagnoses in a national register of patients. Neuroepidemiology 2007;28:150-4. doi:10.1159/000102143

26 Knudsen LB, Olsen J. The Danish Medical Birth Registry. Dan Med Bull 1998;45:320-3.

27 Timm FP, Houle TT, Grabitz SD, et al. Migraine and risk of perioperative ischemic stroke and hospital readmission: hospital based registry study. BMJ 2017;356:i6635. doi:10.1136/bmj.i6635

28 Kurth T, Schürks M, Logroscino G, Gaziano JM, Buring JE. Migraine, vascular risk, and cardiovascular events in women: prospective cohort study. BMJ 2008;337:a636. doi:10.1136/bmj.a636

29 Sacco S, Ornello R, Ripa P, Pistoia F, Carolei A. Migraine and hemorrhagic stroke: a meta-analysis. Stroke 2013;44:3032-8. doi:10.1161/STROKEAHA.113.002465

30 Sacco S, Cerone D, Carolei A. Comorbid neuropathologies in migraine: an update on cerebrovascular and cardiovascular aspects. J Headache Pain 2008:9:237-48, doi:10.1007/s10194-008-0048-4

31 Peng KP, Chen YT, Fuh JL, Tang CH, Wang SJ. Association between migraine and risk of venous thromboembolism: A nationwide cohort study. Headache 2016;56:1290-9. doi:10.1111/head.12885

32 Schwaiger J, Kiechl S, Stockner H, et al. Burden of atherosclerosis and risk of venous thromboembolism in patients with migraine. Neurology 2008;71:937-43. doi:10.1212/01.wnl.0000325918.48056.75

33 Peng KP, Chen YT, Fuh JL, Tang CH, Wang SJ. Association between migraine and risk of venous thromboembolism: A nationwide cohort study. Headache 2016:56:1290-9. doi:10.1111/head.12885

34 Bushnell CD, Jamison M, James AH. Migraines during pregnancy linked to stroke and vascular diseases: US population based casecontrol study. BMJ 2009;338:b664. doi:10.1136/bmj.b664

35 Ungprasert P, Srivali N, Wijarnpreecha K, Charoenpong P, Knight EL. Non-steroidal anti-inflammatory drugs and risk of venous thromboembolism: a systematic review and metaanalysis. Rheumatology (Oxford) 2015;54:736-42. doi:10.1093/ rheumatology/keu408

36 Schmidt M, Christiansen CF, Mehnert F, Rothman KJ, Sørensen HT. Non-steroidal anti-inflammatory drug use and risk of atrial fibrillation or flutter: population based case-control study. BMJ 2011;343:d3450. doi:10.1136/bmj.d3450

37 Roberto G, Raschi E, Piccinni C, et al. Adverse cardiovascular events associated with triptans and ergotamines for treatment of migraine: systematic review of observational studies. Cephalalgia 2015;35:118-31. doi:10.1177/0333102414550416

38 Harriott AM, Barrett KM. Dissecting the association between migraine and stroke. Curr Neurol Neurosci Rep 2015;15:5. doi:10.1007/ s11910-015-0530-8

39 Hippisley-Cox J, Coupland C, Brindle P. Development and validation of QRISK3 risk prediction algorithms to estimate future risk of cardiovascular disease: prospective cohort study. BMJ 2017;357:j2099. doi:10.1136/bmj.j2099

40 National Institute for Health and Care Excellence. Headaches in over 12s: diagnosis and management. 2015. www.nice.org.uk/guidance/ cg150/chapter/recommendations.

41 Holland S, Silberstein SD, Freitag F, Dodick DW, Argoff C, Ashman E, Quality Standards Subcommittee of the American Academy of Neurology and the American Headache Society. Evidence-based guideline update: NSAIDs and other complementary treatments for episodic migraine prevention in adults: report of the Quality Standards Subcommittee of the American Academy of Neurology and the American Headache Society. Neurology 2012;78:1346-53. doi:10.1212/WNL.0b013e3182535d0c

Supplementary: tables 